\title{
Pest species of Coccoidea (Hemiptera; Coccomorpha) in forest of Turkey
}

\author{
Selma Ülgentürk ${ }^{\mathrm{a}, *}$ (i), Özden Dokuyucu ${ }^{\mathrm{a}}$ (i)
}

\begin{abstract}
Forest area of Turkey is 22.6 million hectares, which constitutes $28.8 \%$ of the overall land area. $47 \%$ of the forests is coniferous, $32 \%$ is broad-leaved tree species and $21 \%$ mixed species. The Scale insects (Hemiptera: Coccomorpha) are cryptic and mostly invisible insects which are mostly small less than $5 \mathrm{~mm}$ long. Many scale insects are economically important pests of agriculture, horticulture, and forestry. Around 400 scale insect species are known in Turkey, totally 119 Coccoidea species are fed on forest trees and shrubs; among 45 species of them on conifers and 74 species on broad-leaves trees and shrubs. Marchalina hellenica Gennadius (Marchalinidae) is a famous and economically important species that resourced honeydew honey called "pine honey". This study examines scale insects, their distribution, host plants, natural enemies, damages, and role of forest biodiversity in Turkey.

Keywords: Scale insect, Biodiversity, Conifer, Biology, Damage
\end{abstract}

\section{Türkiye ormanlarında zararlı Coccoidea (Hemiptera; Coccomorpha) türleri}

\begin{abstract}
Özet: Türkiye 22.6 milyon hektar orman alanına sahiptir. Türkiye'nin yüzöçümünün \%28.8'ini kaplayan bu ormanların \%47'sini iğne yapraklılar, \%32'sini geniş yapraklı ağaçlar ve \%21'ini karışık ormanlar oluşturmaktadır. Kabuklubitler (Hemiptera: Coccoidea), saklı yerlerde yaşayan, genellikle 5 mm'den daha küçük olduğu için gözden kaçırılan böceklerdir. Birçok kabuklubit türü tarım ve orman bitkilerinin ekonomik önemde zarar meydana getirmektedir. Türkiye'de varlığı bilinen 400 civarında kabuklubit türünden, 45 tür koniferlerde, 74 tür ise geniş yapraklı ağaç ve çalılarda olmak üzere, toplam 119 Coccoidea türü orman bitkilerinde beslenmektedir. Marchalina hellenica Gennadius (Marchalinidae) bunlardan en bilinen ve ekonomik öneme sahip bir tür olup, "çam balı" olarak bilinen salgıbalına kaynaklık etmektedir. Bu çalışmada Türkiye ormanlarında görülen kabuklubit türlerinin yayılışları, konukçuları, doğal düşmanları, zararları ve orman biyoçeşitliliğindeki rolleri incelenmiştir.

Anahtar kelimeler: Kabuklubitler, Biyoçeşitlilik, Konifer, Biyoloji, Zarar
\end{abstract}

\section{Introduction}

The total forest area of Turkey is 22,342,935 hectares and it consists of $29 \%$ of the country (TOD, 2019). Forests are generally located on mountainous areas and they are usually natural and semi-natural with high biodiversity value. Turkey has 9000 plant species of which 3649 (31.8\%) is endemic (FAO, 2019). Most of these plants are located in forest areas. Approximately 800 woody taxa occur in the country's forests. The predominant species are Pinus brutia Ten., Pinus halepensis Mill., Pinus nigra Arnold, Pinus pinea L., Pinus sylvestris L., Abies spp. [A. cilicica (Ant. and Kotschy), A. nordmannia (Steven)], Picea orientalis (L.), Cedrus libani A. Rich (Pinaceae), Cupressus sempervirens L., Juniperus spp. (Cupressaceae), Fagus orientalis Lipsky, Quercus spp., Castanea sativa Mill. (Fagaceae), Alnus spp. and Carpinus betulus L. (Betulaceae). Main tree species of the Turkey forest are oaks (26.25\%), Turkish pine $(25.13 \%)$, Crimaen pine $(19.02 \%)$, beech $(8.55 \%)$, Scots pine $(6.80 \%)$, juniper $(4.29 \%)$, cedar $(2.16 \%)$, fir $(2.62 \%)$ and spruce trees $(1.45 \%)$. These plants occur as pure stand or mix stand in forests (Anonymous, 2019).

Insects and plant diseases are the important factors that damage forest trees. Scale insects (Hemiptera;
Coccomorpha) feed by sucking the sap of plants from either the phloem or parenchyma and cause reduced host vigor, reduced productivity, defoliation, premature falling of leaves and dieback of branches. In addition, feeding by these insects may cause discoloration and disfiguration and gall formations. They are often cryptic in habit and mostly small size (less than $5 \mathrm{~mm}$ long) therefore undetectable until plant damage is substantial. This point is very important in quarantine and plant protection. There are estimated to be almost 8000 Coccoidea species in the world (Garcia et al., 2016). They are economically important pests of agriculture, horticulture and forest plants (Kosztarab and Kozar, 1988).

\section{Scale insects}

Scale insects have protective covering such as powdery, waxy, and hardy scales. The wax is produced by epidermal glands (mostly cuticular pores, ducts). Scale insects have very specific morphological and biological features. Male scale insects display complete metamorphosis, whereas female is paedomorphic. Female scale insects have often sedentary habit and have four or five biological stages (egg, two or three nymph instars and adult). The female either lays eggs in a cavity under her body or in a waxy sac (ovisac). Crawlers (first instar nymphs) are mobile and play

\footnotetext{
a Ankara Üniversitesi Ziraat Fakültesi Bitki Koruma Bölümü, Dışkapı, Ankara

@ * Corresponding author (İletişim yazarı): ulgentur@agri.ankara.edu.tr

$\checkmark \quad$ Received (Geliş tarihi): 06.09.2019, Accepted (Kabul tarihi): 08.11.2019
}

Citation (Atıf): Ülgentürk, S., Dokuyucu, Ö. 2019. Scale insects in forest plants in Turkey. Turkish Journal of Forestry, 20(4): 482-491. DOI: $10.18182 / \mathrm{tjf} .616353$ 
very important role in dispersing to other plants. Male scale insects have five or six biological stages (egg, two or three nymph stages, prepupa, pupa and adult). Adult males are tiny flies that not feed and live only a few days. Depending on species and the climate, the number of generations is variable between one to seven or eight in a year (Kosztarab and Kozar, 1988). Scale insects on forest plants are studied in Turkey by some authors (Bodenheimer, 1949; 1953; Schmitschek, 1953; Çanakçığlu, 1977; Selmi, 1979; Ülgentürk et al., 2004; Ülgentürk et al., 2012a,b). The number of forest trees used as park trees and new plant species imported as ornamental plants may lead to new insect species to enter the new regions. Insect species which are not cause a serious damage in their natural habitat may reproduce uncontrollably and inflict damage in a new ecosystem as they may not have natural enemies (Şahin and Ülgentürk, 2011). While Torosaspis cedricola (Balachowsky \& Alkan) (Diaspididae), for example, sustaining their existence in low populations in natural cedar forests, it is the major pest of cedar trees in urban greenbelts (Dostbil and Ülgentürk, 2016; Ülgentürk et al., 2012a; Ülgentürk et al., 2019a). The maritime pine bast scale, Matsucoccus feytaudi Ducasse (Matsucoccidae), occurs in the western part of the Mediterranean basin and causes damage in South Eastern France and Italy, where it was accidentally introduced (Foldi, 2004).

Generally, mostly scale insects leave honeydew that covers all parts of the plant, and causing sooty mould. This may be indirectly detrimental to plants by excluding sunlight and interfering with photosynthesis in the leaves as well plants lose their aesthetic and economic value (Kosztarab and Kozar, 1988; Ülgentürk et al., 2019a,b). On the other hand, honeydew is important nutrient for many species such as insects, birds, and also humans. Honeydew is collected and used in producing of honeydew honey by honeybees. For examples honeydew of Marchalina hellenica Gennadius (Marchalinidae) is one of the important source of pine honey in Greece and Turkey (Gürkan, 2005Ülgentürk et al., 2012a ,). Pine honey has economic importance for many beekeepers and for forest villagers, as well. In addition to $M$. hellenica, many other hemipters are known as honeydew producer on cedar, chestnut, oaks, linden, and pine trees in Turkey (Ülgentürk et al., 2012b; Ülgentürk et al., 2013a, b; Ülgentürk et al., 2019b). Some scale insects are also known as the vector of plant diseases (Le Maguet et al., 2012). For example, the beech scale Cryptococcus fagisuga (Douglas) (Eriococcidae) feeds on beech trees (Fagus spp.). Attack by beech scale alone does not seriously damage beech trees; however, the feeding punctures in the bark allow beech bark disease, [Nectria coccinea var. faginata or $N$. galligena (Hypocreales: Nectriaceae)] a serious threat to the timber industry in Europe and in North America, to gain access to the cambium and phloem tissues (Houston, 1994).

Unfortunately, most of the studies which examine mostly host plants and distribution of scale insects occurring on forest areas in Turkey. There is very limited research on their biology, damage and natural enemies in forests. In this study, scale insects that feed on conifers (Table 1) and species that feed on broad-leaved trees and shrubs (Table 2) in Turkey are taken separately. Information of some important species on their biology, damage and natural enemies in urban green areas (forest, parks and gardens) are also given.

Table 1. Scale insects on conifer trees in Turkey

\begin{tabular}{|c|c|c|c|}
\hline Species & Host plants & Distribution $* * *$ & References \\
\hline \multicolumn{4}{|c|}{ Coccidae } \\
\hline Ceroplastes floridensis Comstock & C. libani & $\mathrm{Me}$ & Ülgentürk et al., 2012b \\
\hline Coccus hesperidum Linnaeus & $\begin{array}{l}\text { Abies bornmuelleriana Mattif., Cephalotaxus drupacea } \\
\text { Sieb \& Zucc., Cedrus libani A. Rich, C. atlantica } \\
\text { Manetti, Picea orientalis (L.) Pinus brutia Tenore, } P \text {. } \\
\text { maritima Lam. P. pinea L., P. sylvestris L., Taxus } \\
\text { baccata L. }\end{array}$ & $\mathrm{Ae}, \mathrm{Bs}, \mathrm{Ma}, \mathrm{Me}$ & $\begin{array}{l}\text { Çanakçıŏlu, 1977; Ülgentürk } \\
\text { and Çanakçığlu, } 2004\end{array}$ \\
\hline C. pseudomagnoliarum (Kuwana) & P. sylvestris & $\mathrm{Bs}, \mathrm{Ca}$ & Çanakçıŏglu, 1977 \\
\hline Nemolecanium abietis Borchsenius & A. bornmuelleriana, A. nordmanniana (Steven) & $\mathrm{Bs}, \mathrm{Ca}$ & Ülgentürk et al., 2019a \\
\hline Nemolecanium aptii (Bodenheimer) & A. nordmanniana & Bs & Bodenheimer, 1953 \\
\hline Eulecanium sericeum Lindinger & Abies cilicica (Antoine \& Kotschy), A. bornmuelleriana & Bs, Sea & Ülgentürk et al., 2019b \\
\hline $\begin{array}{l}\text { Physokermes hellenicus Kozár and } \\
\text { Gounari }\end{array}$ & A. bornтиelleriana, A. cilicica & $\mathrm{Bs}, \mathrm{Ca}, \mathrm{Me}$ & $\begin{array}{l}\text { Ülgentürk, 2016; } \\
\text { Ülgentürk et al., 2019b }\end{array}$ \\
\hline Physokermes piceae (Schrank) & $\begin{array}{l}\text { Picea abies }(\mathrm{L} .), P \text {. pungens Engelm., P. pungens var. } \\
\text { glauca Beissn., P. orientalis }\end{array}$ & $\mathrm{Bs}, \mathrm{Ca}, \mathrm{Ma}$ & $\begin{array}{l}\text { Canakçıoğlu, 1977; Selmi, 1979; } \\
\text { Ülgentürk and Özdemir, } 2019\end{array}$ \\
\hline Poaspis intermedia Goux & P. brutia & Мa & Ülgentürk et al., 2012c \\
\hline Pulvinaria floccifera (Westwood) & Taxus baccata L., T. baccata var. fastigiata & $\mathrm{Bs}, \mathrm{Ma}$ & $\begin{array}{l}\text { Bodenheimer, 1953; Selmi, 1979; } \\
\text { Ülgentürk et al., } 2008\end{array}$ \\
\hline \multicolumn{4}{|c|}{ Diaspididae } \\
\hline Aonidia mediterranea Lindinger & $\begin{array}{l}\text { Cupressus sempervirens L., Juniperus sp. Pinus sp., P. } \\
\text { brutia }\end{array}$ & $\mathrm{Me}, \mathrm{Ea}$ & Yaşar, 1995; Kaydan et al., 2013 \\
\hline Aspidiotus nerii (Bouché) & C. libani, P. abies, T. baccata & $\mathrm{Ma}, \mathrm{Me}$ & Selmi, 1979 \\
\hline Chionaspis kabyliensis Balachowsky & C. libani & $\mathrm{Me}$ & Ülgentürk et al., 2012b \\
\hline Carulaspis juniperi (Bouché) & $\begin{array}{l}\text { Platyclaudus orientalis (L.), Calocedrus decurrens } \\
\text { (Torrey), Chaemaecyparis lawsoniana (A. Murray), } \\
\text { Cupressus sp., C. arizonica Greene, Juniperus sabina } \\
\text { var. tamariscifolia Ait. }\end{array}$ & $\mathrm{Ae}, \mathrm{Ca}, \mathrm{Me}$ & $\begin{array}{l}\text { Kaydan et al., 2013; Ülgentürk et } \\
\text { al., 2019a }\end{array}$ \\
\hline
\end{tabular}


Table 1. Scale insects on conifer trees in Turkey (continued)

\begin{tabular}{|c|c|c|c|}
\hline Species & Host plants & Distribution $* * *$ & References \\
\hline Carulaspis minima (Signoret) & $\begin{array}{l}\text { Arceuthos drupacea Ant. \& Kotschy, B. orientalis C. } \\
\text { lawsonia, C.decurrens, Cupressus spp., C. sempervirens } \\
\text { L., C. sempervirens var. horizontalis (Mill.), C. } \\
\text { sempervirens var. pyramidalis Nym., C. goveniana } \\
\text { (Gordon), C. arizonica Juniperus oxycedrus L., J. } \\
\text { communis L., J. foetidissima Willd., J. sabina L., J. } \\
\text { sabina var. nana Syme., J. excelsa, P. orientalis }\end{array}$ & $\mathrm{Ae}, \mathrm{Bs}, \mathrm{Ma}, \mathrm{Me}$ & $\begin{array}{l}\text { Bodenheimer, 1949, 1952; } \\
\text { Kaydan et al., } 2013\end{array}$ \\
\hline Diaspidiotus jaapi Leonardi & C. libani, P. brutia & $\mathrm{Ae}$ & Ülgentürk et al., 2012b \\
\hline $\begin{array}{l}\text { Chrysomphalus dictyospermi } \\
\text { (Morgan) }\end{array}$ & T. baccata & $\mathrm{Ma}$ & Selmi, 1979 \\
\hline Dynaspidiotus britannicus (Newstead) & $\begin{array}{l}\text { A. bornmuelleriana, A. equi-trojani Ascher \& Sint., } C \text {. } \\
\text { atlanticus, C. libani, P. pungens }\end{array}$ & Bs, Ca, Ma & $\begin{array}{l}\text { Selmi, 1979; Ülgentürk et al., } \\
\text { 2019a }\end{array}$ \\
\hline Dynaspidiotus abietis (Schrank) & Abies sp., Pinus sp. & $\mathrm{Bs}, \mathrm{Ca}, \mathrm{Me}, \mathrm{Ma}$ & $\begin{array}{l}\text { Özkazanç and Yücel, 1985; } \\
\text { Yaşar, } 1995\end{array}$ \\
\hline Dynaspidiotus abieticola (Koreneos) & $\begin{array}{l}\text { A. bornmuelleriana, A. concolor (Gord.), A. equi- } \\
\text { trojani, C. libani, P. pungens }\end{array}$ & $\mathrm{Ca}, \mathrm{Me}$ & $\begin{array}{l}\text { Yaşar, 1995; Ülgentürk et al., } \\
\text { 2012b; }\end{array}$ \\
\hline Gomezmenoraspis pinicola Leonardi & P. brutia, $P$. pinea, $P$. sylvestris & $\mathrm{Ae}, \mathrm{Bs}, \mathrm{Me}, \mathrm{Ma}$ & $\begin{array}{l}\text { Ülgentürk et al., 2012b; Kaydan } \\
\text { et al., } 2014\end{array}$ \\
\hline $\begin{array}{l}\text { Gomezmenoraspis nr pinicola } \\
\text { Leonardi }\end{array}$ & C. libani & $\mathrm{Me}$ & Ülgentürk et al., 2012c \\
\hline Leucapis loewi Colvée & $\begin{array}{l}\text { Pinus sp., } P . \text { halepensis Mill., } P \text {. nigra, } P \text {. nigra } \\
\text { subsp.pallasiana, } P \text {. pinea, } P \text {. sylvestris }\end{array}$ & $\begin{array}{l}\text { Ae, Bs, Ca, Ea, } \\
\text { Me, Ma }\end{array}$ & $\begin{array}{l}\text { Çanakçığlu, 1977; Selmi, 1979; } \\
\text { Ülgentürk et al., 2004, 2019a }\end{array}$ \\
\hline Leucaspis knemion Hoke & Pinus sp., P. brutia & $\mathrm{Ma}, \mathrm{Me}$ & $\begin{array}{l}\text { Bodenheimer, 1952; Ülgentürk et } \\
\text { al., 2012b; } 2019\end{array}$ \\
\hline L. pini Harting & C. libani, P. brutia, P. halapennis, P. nigra P. pinea & $\mathrm{Ae}, \mathrm{Bs}, \mathrm{Me}, \mathrm{Ma}$ & $\begin{array}{l}\text { Bodenheimer, 1952; Ülgentürk et } \\
\text { al., 2012b,c; } 2019\end{array}$ \\
\hline L. pusilla Löw & $\begin{array}{l}P . \text { brutia, } P \text {. elderica Medv., } P \text {. halepensis, } P \text {. maritima, } \\
P . \text { nigra, } P \text {. nigra subsp. pallasiana, } P \text {. pandarosa } \\
\text { Dougl., } P \text {. pinea, } P \text {. radiate D.Don., } P \text {. roxburghii } \\
\text { Sargenk, } P \text {. sylvestris, } P \text {. strobe L., }\end{array}$ & $\mathrm{Ae}, \mathrm{Ca}, \mathrm{Me}, \mathrm{Ma}$ & $\begin{array}{l}\text { Bodenheimer, 1949,1952; } \\
\text { Çanakçıŏlu, 1977; Selmi, 1979; } \\
\text { Kaydan et al., 2014; Ülgentürk et } \\
\text { al., 2012b,c; } 2019\end{array}$ \\
\hline Lepidosaphes newsteadi (Sulc) & $\begin{array}{l}\text { Abies sp., A. bornmülleriana, } A \text {. pinsapo Boiss., } P \text {. } \\
\text { pungens, } P \text {. nigra }\end{array}$ & $\mathrm{Bs}, \mathrm{Ca}, \mathrm{Ma}$ & Kaydan et al., 2013, 2014 \\
\hline Lepidosaphes juniperi Lindinger & $\begin{array}{l}\text { Juniperus sp., J. excelsa Bieb., Cedrus libani, Pinnus } \\
\text { nigra, P. brutia, T. occidentalis }\end{array}$ & $\mathrm{Ae}, \mathrm{Bs}, \mathrm{Ca}$ & $\begin{array}{l}\text { Kaydan et al., 2013; Ülgentürk et } \\
\text { al., 2019a }\end{array}$ \\
\hline Lepidosaphes ulmi Linneaus & P. brutia & $\mathrm{Ae}$ & Ülgentürk et al., 2012a \\
\hline Lineaspis striata (Newstead) & $\begin{array}{l}\text { Arceuthobium spp., Cupresuss sp. C. sempervirens, } \\
\text { Thuja spp., T. occidentalis }\end{array}$ & $\mathrm{Ca}, \mathrm{Me}$ & $\begin{array}{l}\text { Yaşar, 1995; Ülgentürk et al., } \\
\text { 2019a }\end{array}$ \\
\hline $\begin{array}{l}\text { Torosaspis farsianus (Balachowsky \& } \\
\text { Kaussari) }\end{array}$ & $\begin{array}{l}\text { C.arizonica, C. sempervirens var. horizontalis, } C . \\
\text { sempervirens var. pyramidalis }\end{array}$ & $\mathrm{Me}$ & Keçe Çalışkan and Ulusoy, 2017 \\
\hline T. cedricola Balachowski \& Alkan) & $\begin{array}{l}\text { C. atlantica Manetti, C. atlantica var. glauca Carr., C. } \\
\text { deodora (Roxburg), Cedrus libani }\end{array}$ & $\begin{array}{l}\text { Ae, Bs, Ca, Ea, } \\
\text { Me, Ma, Sea }\end{array}$ & $\begin{array}{l}\text { Yaşar 1995; Ülgentürk et al., } \\
2012 b, 2019 a \text {; Kaydan et al., } \\
2014\end{array}$ \\
\hline \multicolumn{4}{|c|}{ Eriococcidae } \\
\hline Uhleria araucariae Maskell & Araucaria sp. & $?$ & Kozar et al., 2013 \\
\hline \multicolumn{4}{|l|}{ Marchalinidae } \\
\hline Marchalina caucasica Hadzibejli &. nordmanniana, Piceae orientalis & Bs & \multirow{2}{*}{$\begin{array}{l}\text { Ülgentürk et al., 2019b } \\
\text { Bodenheimer, 1953; Ülgentürk et } \\
\text { al., 2012a; 2013a }\end{array}$} \\
\hline Marchalina hellenica Gennadius & .. libani, P. brutia, P. halepensis, P. pinea & $\mathrm{Ae}, \mathrm{Bs}, \mathrm{Me}, \mathrm{Ma}$ & \\
\hline \multicolumn{4}{|c|}{ Matsucoccidae } \\
\hline $\begin{array}{l}\text { Matsucoccus josephi Bodenheimer \& } \\
\text { Harpaz }\end{array}$ & Pinus spp., P. brutia, $P$. pinea & Ae, Ma, Me & $\begin{array}{l}\text { Ülgentürk et al., 2012c; } \\
\text { Ülgentürk et al., } 2016\end{array}$ \\
\hline M. pini Green & Pinus sylvestris & $\mathrm{Ca}$ & Ülgentürk et al., 2019a \\
\hline \multicolumn{4}{|c|}{ Monophlebidae } \\
\hline Palaecoccus fuscipennis Burnmeister & Pinus sp. P. brutia, $P$. pinea & $\mathrm{Ae}, \mathrm{Ma}, \mathrm{Me}$ & $\begin{array}{l}\text { Bodenheimer, 1953; Ülgentürk et } \\
\text { al., 2012c }\end{array}$ \\
\hline \multicolumn{4}{|c|}{ Pseudococcidae } \\
\hline $\begin{array}{l}\text { Phenacoccus arambourgi } \\
\text { Balachowsky }\end{array}$ & C. libani & $\mathrm{Me}$ & Ülgentürk et al., 2012b \\
\hline Phenacoccus piceae Löw & P. orientalis & Bs & Ülgentürk and Özdemir, 2019 \\
\hline Phenacoccus yerushalmi Ben-Dov & P. brutia, P. sylvestris & $\mathrm{Ae}, \mathrm{Me}$ & $\begin{array}{l}\text { Ben-Dov et al., 2006; Ülgentürk } \\
\text { et al., 2012c }\end{array}$ \\
\hline Planococcus vovae (Nasonov) & $\begin{array}{l}\text { Cupressus sp. C. sempervirens, } \text { C. sempervirens var. } \\
\text { horizontalis, C. sempervirens var. pyramidalis, } C . \\
\text { goweniana, Juniperis excels Bieb., Libocedrus } \\
\text { decurrens Torr., T. baccata, Thuja occidentalis L. }\end{array}$ & $\begin{array}{l}\mathrm{Ae}, \mathrm{Bs}, \mathrm{Ca}, \mathrm{Me}, \\
\mathrm{Ma},\end{array}$ & $\begin{array}{l}\text { Düzgüneş, 1982; Selmi, 1979; } \\
\text { Ülgentürk et al., 2013b }\end{array}$ \\
\hline Pseudococcus viburni (Signoret) & C.drupacea & $\mathrm{Ma}$ & Selmi, 1979 \\
\hline
\end{tabular}

***Aegean (Ae), Blacksea (Bs), Mediterranean (Me), Marmara (Ma), Central Anatolia (Ca), Eastern Anatolia (Ea) and Southeastern Anatolia (Sea) 
Table 2. Scale insects on broad-leaved trees, shrubs and other plants in Turkey

\begin{tabular}{|c|c|c|c|}
\hline Species & Host plants & $\begin{array}{l}\text { Distribution } \\
* * *\end{array}$ & References \\
\hline \multicolumn{4}{|c|}{ Acanthococcidae } \\
\hline Acanthococcus aceris Signoret & Quercus sp., Platanus orientalis L. & $\mathrm{Ea}$ & Kaydan and Kozár, 2008 \\
\hline A. melnikensis Hodgson and Trencheva & Myrtus communis L. & $\mathrm{Ae}$ & Ülgentürk et al., 2013b \\
\hline A. roboris Goux & Castanea sp., Quercus sp., Q. cerris L., Q. suber L. & $\mathrm{Ae}, \mathrm{Bs} \mathrm{Me}$ & $\begin{array}{l}\text { Kosztarab and Kozár, 1988; } \\
\text { Kaydan and Kozar, } 2008\end{array}$ \\
\hline A.salicis (Borchsenius) & Salix alba L. & $\mathrm{Ea}$ & Kozár et al.. 2013 \\
\hline Gossyparia spurius (Modeer) & Quercus sp., Ulmus spp., Viscum album L. & $\mathrm{Ae}, \mathrm{Ca}, \mathrm{Ma}$ & $\begin{array}{l}\text { Çanakçıoğlu, 1977; Ülgentürk et } \\
\text { al., } 2003\end{array}$ \\
\hline Kotejacoccus turcicus Kaydan \& Kozár & Quercus sp., & $\mathrm{Ea}$ & Kaydan and Kozár, 2008 \\
\hline $\begin{array}{l}\text { Neoacanthococcus atlihani Kaydan \& } \\
\text { Kozár }\end{array}$ & Tamarix sp. & 2 & Kaydan and Kozár, 2010 \\
\hline Orontesicoccus lauri (Erkılıç) & Laurus nobilis L. & $\mathrm{Me}$ & Erkılıç et al., 2011 \\
\hline \multicolumn{4}{|c|}{ Asterolecaniidae } \\
\hline Asterodiaspis bella (Russell) & Quercus sp., Q. robor L. & $\mathrm{Ca}, \mathrm{Ea}$, & $\begin{array}{l}\text { Zeki et al. 2005; Ülgentürk et } \\
\text { al., 2013a }\end{array}$ \\
\hline A. hadzibeyliae Borchsenius & Quercus sp. & $\mathrm{Ea}$ & Ülgentürk et al., 2013a \\
\hline A. ilicicola (Targioni Tozzetti) & Quercus sp., Q. coccifera L. & $\mathrm{Ae}, \mathrm{Me}$ & $\begin{array}{l}\text { Bodenheimer, 1953; Ülgentürk } \\
\text { et al., 2013a }\end{array}$ \\
\hline A. mina (Russell) & Qercus sp. & $\mathrm{Bs}, \mathrm{Ea}$ & $\begin{array}{l}\text { Kaydan et al., 2013; Ülgentürk } \\
\text { et al., 2013a }\end{array}$ \\
\hline A. minus Lindinger & Q. coccifera & $\mathrm{Ca}, \mathrm{Ma}, \mathrm{Me}$ & Bodenheimer, 1953 \\
\hline A.quercicola (Bouché) & Quercus sp., Q. brantii Lindl., Q. robor & $\mathrm{Ca}, \mathrm{Me}$ & $\begin{array}{l}\text { Zeki et al., 2005; Ülgentürk et } \\
\text { al., 2013a }\end{array}$ \\
\hline A. repugnans (Russell) & Quercus sp. & $\mathrm{Bs}, \mathrm{Ea}$ & $\begin{array}{l}\text { Ülgentürk et al., 2013a; Kaydan } \\
\text { et al., } 2014\end{array}$ \\
\hline A. variolasa (Ratzeburg) & $\begin{array}{l}\text { Quercus sp., Q. aegilops L., Q. coccifera, Q. robor v. } \\
\text { fastiata }\end{array}$ & $\begin{array}{l}\mathrm{Ae}, \mathrm{Ca}, \mathrm{Me}, \\
\mathrm{Ma}\end{array}$ & $\begin{array}{l}\text { Yaşar, 1991; Ülgentürk et al., } \\
\text { 2013a }\end{array}$ \\
\hline \multicolumn{4}{|c|}{ Coccidae } \\
\hline Ceroplastes floridensis Comstock ** & Arbutus unedo L., L. nobilis, M. communis & $\mathrm{Ae}, \mathrm{Me}$ & $\begin{array}{l}\text { Bodenheimer, 1953; Ülgentürk } \\
\text { et al., } 2013 \mathrm{~b}\end{array}$ \\
\hline C. japonica Green* & $\begin{array}{l}\text { Acer negundo L., A. pseudoplatanus L., Aesculus } \\
\text { hippocastanum L., Morus alba L., Nerium oleander L., } \\
\text { Pistaciae terebinthus L., L. nobilis, Ulmus sp. }\end{array}$ & $\mathrm{Ma}$ & Ülgentürk et al., 2008 \\
\hline Ceroplastes rusci (Linnaeus) ${ }^{* *}$ & $\begin{array}{l}\text { 1. communis, } N \text {. oleander, P. palaestina Boiss, P. vera L., } \\
\text { 'opulus sp., Olea europea L., Salix sp. }\end{array}$ & $\mathrm{Ae}, \mathrm{Bs}$ & $\begin{array}{l}\text { Kaydan et al., 2013, 2014; } \\
\text { Ülgentürk et al., 2013b }\end{array}$ \\
\hline Coccus hesperidum L.** & $\begin{array}{l}\text { l.pseudoplatanus, Crateagus monogyna Jacq, L. nobilis, } \\
\text { onicera caprifolium, N. oleander, P. atlantica, Quercus } \\
\text { pp., }\end{array}$ & $\mathrm{Ma}$ & $\begin{array}{l}\text { Bodenheimer, 1953; Ülgentürk } \\
\text { et al., } 2008\end{array}$ \\
\hline Eulecanium ciliatum (Douglas)* & $\begin{array}{l}\text { Quercus sp., Q. penduculiflora K. Koch, Jasminum } \\
\text { fruticans L., Acer campestre L., A. pseudoplatanus, C. } \\
\text { monogyna, C. oxycantha L., Ribes sp., Cydonia sp., Malus } \\
\text { sp. }\end{array}$ & $\mathrm{Ca}$ & $\begin{array}{l}\text { Bodenheimer, 1953; Ülgentürk } \\
\text { and Toros, 1999a; Zeki et al., } \\
2005\end{array}$ \\
\hline Eulecanium cerasorum (Cockerell)* & $Q$. robur & $\mathrm{Ca}$ & Ülgentürk et al., 2013a \\
\hline Eulecanium tiliae (Linnaeus) & $\begin{array}{l}\text { A. pseudoplatanus, A. hippocastanum, Crateagus sp., } N \text {. } \\
\text { oleander, Quercus sp., Q. robur, Q. cercis, } Q \text {. } \\
\text { penduculiflora K. Koch, Ulmus minor Mill. (As } U . \\
\text { campestre), Tilia } \mathrm{sp.}\end{array}$ & $\mathrm{Bs}, \mathrm{Ma}, \mathrm{Ca}$ & $\begin{array}{l}\text { Bodenheimer, 1953; Ülgentürk } \\
\text { and Toros, 1999a; Ülgentürk et } \\
\text { al., 2013b; Zeki et al., } 2005\end{array}$ \\
\hline Filippia follicularis (Targioni-Tozzetti) & $\begin{array}{l}\text { Fraxinus americana L., F. excelsior L., Jasminium sp., } \\
\text { Olaea sp. Olea europea L., Phillyrea sp., Prunus avium } \\
\text { L., P. dulcis (Mill), Quercus sp. Viburnum sp., V.m }\end{array}$ & $\begin{array}{l}\mathrm{Ae}, \mathrm{Bs}, \mathrm{Ca} \text {, } \\
\mathrm{Me}\end{array}$ & $\begin{array}{l}\text { Bodenheimer, 1953; Ülgentürk } \\
\text { and Toros, 1999a; Ülgentürk et } \\
\text { al., 2013b }\end{array}$ \\
\hline Lichtensia viburni Signoret & $\begin{array}{l}\text { Ceratonia silique L., Hedera helix L., Phillyrea sp., } \\
\text { Pistaciae sp., Viburnum tinus L. }\end{array}$ & $\mathrm{Ae}$ & $\begin{array}{l}\text { Yaşar, 1990; Ülgentürk et al., } \\
\text { 2008; }\end{array}$ \\
\hline Neopulvinaria innumerabilis Rathorn* & $\begin{array}{l}\text { Acer negundo, Catalpa bignonioides WalterC. monogyna, } \\
\text { Gleditsia triacanthos L., Morus alba, Parthenocissus } \\
\text { quinquefolia (L.), P.identalis, Quercus sp., Robinia } \\
\text { pseudoacacia L., Spirea } \text { sp., Tilia } \text { sp., Vitis vinifera }\end{array}$ & $\mathrm{Ca}, \mathrm{Ma}$ & Ülgentürk and Ayhan, 2011 \\
\hline Parthenolecanium corni (Bouche)* & $\begin{array}{l}\text { Crateagus sp., Corylus avellana L., Fagus sp., Fraxinus } \\
\text { sp., M. alba, Quercus sp., Salix sp., Ulmus sp. }\end{array}$ & $\begin{array}{l}\mathrm{Bs}, \mathrm{Ca}, \mathrm{Me} \\
\mathrm{Ma}\end{array}$ & $\begin{array}{l}\text { Bodenheimer, 1953; Ülgentürk } \\
\text { and Toros, 1999a, Kaydan et al., } \\
2014\end{array}$ \\
\hline P. rufulum (Cockerell) & C. avellana, Quercus sp. & $\begin{array}{l}\mathrm{Ae}, \mathrm{Bs}, \mathrm{Ca}, \\
\mathrm{Ma}\end{array}$ & $\begin{array}{l}\text { Kaydan et al., 2013; Kaplan and } \\
\text { Turanlı, } 2016\end{array}$ \\
\hline $\begin{array}{l}\text { Parthenolecanium tamaricis } \\
\text { (Bodenheimer) }\end{array}$ & Tamarix pallasi Desv. & $\mathrm{Ca} ?$ & Bodenheimer, 1953 \\
\hline Pulvinaria floccifera (Westwood) & Prunus laurocerasus L., Camellia sinensis (L.) & Bs, Ma & $\begin{array}{l}\text { Alkan, 1957; Ülgentürk et al., } \\
2008\end{array}$ \\
\hline P. terrestris Borchsenius & Crateagus sp. & & Kaydan et al., 2013 \\
\hline Pulvinaria vitis (Linnaeus) & $\begin{array}{l}\text { Crateagus sp., Populus nigra L., P. tremula L., Quercus } \\
\text { sp., Salix sp., S. alba, Ulmus sp. }\end{array}$ & $\begin{array}{l}\mathrm{Ae}, \mathrm{Bs}, \mathrm{Ca} \\
\mathrm{Ea}, \mathrm{Ma}, \mathrm{Me}\end{array}$ & Kaydan et al., 2013 \\
\hline $\begin{array}{l}\text { Rhodococcus perornatus (Cockerell \& } \\
\text { Parrott) }\end{array}$ & $\begin{array}{l}\text { Rosa sp., R. cinnamomea L., R. canina L., R. damascena } \\
\text { Mill., Rosa pimpinellifolia L. }\end{array}$ & $\mathrm{Ca}, \mathrm{Me}$ & Zeki et al., 2005 \\
\hline Saissetia oleae (Olivier) & A. unedo, Phillyrea sp., O. europea, N. oleander & $\begin{array}{l}\text { Ae, Bs, Ma, } \\
\text { Me }\end{array}$ & Kaydan et al., 2013, 2014 \\
\hline
\end{tabular}


Table 2. Scale insects on broad-leaved trees, shrubs and other plants in Turkey (continued)

\begin{tabular}{|c|c|c|c|}
\hline Species & Host plants & $\begin{array}{l}\text { Distribution } \\
* * *\end{array}$ & References \\
\hline \multicolumn{4}{|c|}{ Cryptococcidae } \\
\hline Cryptococcus fagisuga Lindinger & F. orientalis & Bs & Kozár et al. 2013 \\
\hline Pseudochermes fraxini (Kaltenbach) & F. excelsior & Ma & $\begin{array}{l}\text { Bodenheimer, 1953; Kozár et } \\
\text { al., } 2013\end{array}$ \\
\hline \multicolumn{4}{|c|}{ Diaspididae } \\
\hline Aonidia lauri (Bouche) & L. nobilis & Ma Me, Sea & Kaydan et al., 2013 \\
\hline Aonidiella aurantii (Maskell) & Cerotonia siliqua L., L. nobilis & $\mathrm{Me}$ & Kaydan et al., 2013, 2014 \\
\hline Aspidiotus hedericola Leonardi & L. nobilis, H. helix & Ae, $\mathrm{Me}, \mathrm{Ma}$ & Kaydan et al., 2013 \\
\hline A. nerii Bouché & $\begin{array}{l}\text { Acacia cultiformis G. Donn., A. cyanophilla Lindley, } \\
\text { Aucuba japonica Tunb., H. helix, Jasminum sp., L. nobilis }\end{array}$ & $\begin{array}{l}\text { Ae, Bs, Me, } \\
\text { Ma }\end{array}$ & Kaydan et al., 2013, 2014 \\
\hline Chionaspis etrusca Leonardi & Tamarix sp. & $\mathrm{Ca}, \mathrm{Ea}, \mathrm{Me}$ & Bodenheimer, 1953 \\
\hline Chionaspis salicis (Linnaeus) & $\begin{array}{l}\text { P. alba, P.x canadensis Moench, } P \text {. nigra L., P. tremula, } \\
\text { Salix alba, S. babylonica L., Ulmus sp. }\end{array}$ & Bs, Ca, Ea, & Kaydan et al., 2013, 2014 \\
\hline C. lepineyi Balachowsky & Quercus sp. & $\mathrm{Ea}$ & Kaydan et al., 2013, 2014 \\
\hline $\begin{array}{l}\text { Chrysomphalus dictyospermi } \\
\text { (Morgan)** }\end{array}$ & $\begin{array}{l}\text { B. sempervirens, } C \text {. siliqua, } H \text {. helix, Ilex aquifolium } \mathrm{L} . \text {, } \\
\text { Jasminum sambac (L.), L. nobilis, } M \text { sylvestris, } M . \\
\text { communis, } N \text {. oleander }\end{array}$ & $\mathrm{Ae}, \mathrm{Ma}$ & $\begin{array}{l}\text { Yaşar, 1995; Erözmen and } \\
\text { Yaşar, } 2018\end{array}$ \\
\hline $\begin{array}{l}\text { Comstockaspis perniciosus } \\
\text { (Comstock)** }\end{array}$ & $\begin{array}{l}\text { Crateagus orientalis M.Bieb, Prunus spinose L., Spartium } \\
\text { junceum L. }\end{array}$ & $\begin{array}{l}\mathrm{Bs}, \mathrm{Ca}, \mathrm{Ea}, \\
\mathrm{Me}\end{array}$ & $\begin{array}{l}\text { Kaydan et al., 2013, 2014; } \\
\text { Kaydan et al., 2013, } 2014\end{array}$ \\
\hline Diaspidiotus armenicus (Borchsenius) & P. alba, P.nigra, Salix sp., Ulmus sp. & $\mathrm{Ea}$ & Kaydan et al., 2013 \\
\hline D.caucasicus (Borchsenius) & $\begin{array}{l}\text { Populus sp., P. nigra var. pyramidilis, Quercus sp., Salix } \\
\text { sp. }\end{array}$ & $\mathrm{Bs}, \mathrm{Ca}$ & Kaydan et al., 2013 \\
\hline D.gigas (Thiem\& Gerneck) & Populus nigra, Salix sp. & Bs, Ea, Ma & Kaydan et al., 2013, 2014 \\
\hline D. lenticularis (Lindinger) & Prunus avium $\mathrm{L}$. & Ma & Kaydan et al., 2013 \\
\hline D.kaussarii Balachowsky & Fraxinus sp., M. sylvestris, P.orientalis & $\mathrm{Ea}$ & Kaydan et al., 2013, 2014 \\
\hline D. marani (Zahradnik) & $\begin{array}{l}\text { Fraxinus sp., M. sylvestris, } P \text {. orientalis, } P \text {. avium, Ulmus } \\
\text { americana L. }\end{array}$ & $\begin{array}{l}\mathrm{Bs}, \mathrm{Ca}, \mathrm{Ea} \\
\mathrm{Ma}, \mathrm{Sea}\end{array}$ & Kaydan et al., 2013 \\
\hline D. ostreaeformis (Curtis) & $\begin{array}{l}\text { A. negundo, C. avellana, Liquidambar orientalis Miller, } M \text {. } \\
\text { sylvestris, Populus sp., P. x. canadesis-sp. }\end{array}$ & $\begin{array}{l}\mathrm{Ae}, \mathrm{Bs}, \mathrm{Ca} \\
\mathrm{Ea}, \mathrm{Ma}, \mathrm{Sea}\end{array}$ & Kaydan et al., 2013 \\
\hline D. osborni (Newell \& Cocckerell) & Salix sp. & Sea & Kaydan et al., 2013 \\
\hline D. pyri (Lichtenstein) & Salix sp. & $\begin{array}{l}\mathrm{Bs}, \mathrm{Ca}, \mathrm{Ea}, \\
\mathrm{Ma}, \mathrm{Sea}\end{array}$ & Kaydan et al., 2013 \\
\hline D. uvae (Comstock) & $\begin{array}{l}\text { Celtis siliquasrum L., F. excelsior, P. orientalis, } \\
\text { Paulownia tomentosa (Thunb.) Jacaranda mimosifolia } \\
\text { D.Don, R. pseudoacacia }\end{array}$ & $\mathrm{Me}$ & $\begin{array}{l}\text { Keçe- Çalışkan and Ulusoy, } \\
2017\end{array}$ \\
\hline D. wuenni (Lindinger) & Alnus sp., Quercus sp., & $\mathrm{Ea}$ & Kaydan et al., 2013 \\
\hline D.zonatus (Frauenfeld) & $\begin{array}{l}\text { F. orientalis, Quercus sp., Q. coccifera, Salix sp., Ulmus } \\
\text { americana }\end{array}$ & $\mathrm{Bs}, \mathrm{Ca}, \mathrm{Ea}$ & Kaydan et al., 2013; 2014 \\
\hline Dynaspidiotus britannicus (Newstead) & C.siliqua, Daphne sp., H. helix., L. nobilis, M. communis & $\mathrm{Bs}, \mathrm{Ca}, \mathrm{Me}$ & $\begin{array}{l}\text { Bodenheimer, 1953; Ülgentürk } \\
\text { et al., 2012a; } 2019\end{array}$ \\
\hline Epidiaspis leperii (Signoret) & $\begin{array}{l}\text { A. hippocastaneum, Pistacia sp., Prunus sp., } R . \\
\text { pseudoacacia, Malus sp., Pyrus communis L. }\end{array}$ & $\begin{array}{l}\mathrm{Ae}, \mathrm{Bs}, \mathrm{Ca} \\
\mathrm{Ma}\end{array}$ & $\begin{array}{l}\text { Kaydan et al., 2009; Erözmen } \\
\text { and Yaşar, } 2018\end{array}$ \\
\hline Gonaspidiotus minimus (Leonardi) & Quercus coccifera, $Q$. dschrochensis, $Q$. ilex, Thuja sp. & $\mathrm{Ae}, \mathrm{Me}$ & Bodenheimer, 1952 \\
\hline Lepidosaphes granati Koroneos & Quercus sp., P. orientalis, Ulmus sp., U. glabra & $\mathrm{Ea}$ & Kaydan et al., 2009 \\
\hline Lepidosaphes malicola Borchsenius & $\begin{array}{l}\text { A. negundo, Farxinus excelsior, Populus sp., Quercus sp., } \\
\text { Salix sp. }\end{array}$ & $\mathrm{Ea}$ & Kaydan et al., 2013 \\
\hline L. ulmi (Linnaeus)** & $\begin{array}{l}\text { Acer sp., A. negundo, Fagus sp., Gleditschia sp., Ilex sp., } \\
\text { Populus } \mathrm{sp} ., \text { Quercus sp., Rosa } \mathrm{sp} ., \text { R. canina, } R . \\
\text { damescana, Salix sp., S. junceum }\end{array}$ & $\begin{array}{l}\mathrm{Bs}, \mathrm{Ca}, \mathrm{Ea}, \\
\mathrm{Ma}\end{array}$ & $\begin{array}{l}\text { Kaydan et al. 2013; Erözmen } \\
\text { and Yaşar, } 2018\end{array}$ \\
\hline Melanaspis inopinata (Leonardi) & $\begin{array}{l}\text { A.hippocastaneum, A. unedo, Bauhinia sp., C. } \\
\text { siliquastrum, Fraxinus sp., P.avium, Ulmus sp. }\end{array}$ & $\mathrm{Ca}, \mathrm{Ma}$ & $\begin{array}{l}\text { Bodenheimer, 1949; Ülgentürk } \\
\text { and Toros, 1996; Ayten and } \\
\text { Ülgentürk, } 2007\end{array}$ \\
\hline Parlatoria oleae (Colvée) ${ }^{* *}$ & $\begin{array}{l}\text { Eriobotrya sp., Fraxinus spp., Rosa sp., M. sylvestris, } \\
\text { Prunus sp., R. pseudoacacia }\end{array}$ & $\begin{array}{l}\mathrm{Ae}, \mathrm{Ca}, \mathrm{Me}, \\
\mathrm{Ma}\end{array}$ & Kaydan et al., 2013 \\
\hline P. pergandii (Comstock)** & $\begin{array}{l}\text { C. siliqua, L. nobilis } \\
\text { Aesculus carnea } \text { Briotii, C. bignonioides, Cornus alba L., }\end{array}$ & $\mathrm{Me}, \mathrm{Ae}$ & Kaydan et al., 2013 \\
\hline $\begin{array}{l}\text { Pseudaulacaspis pentagona (Targioni- } \\
\text { Tozzetti)** }\end{array}$ & $\begin{array}{l}\text { Forsythia } x \text { intermedia, F. americana, F. excelsior, M. } \\
\text { alba, Prunus sp., Paulownia tomentosa (Thunb.), P. avium, } \\
\text { P. cerasus, Quercus sp., Rhus typhina L., S.babylonica, S., } \\
\text { Syringia vulgaris L. }\end{array}$ & $\begin{array}{l}\mathrm{Ae}, \mathrm{Bs}, \mathrm{Ca} \\
\mathrm{Ea}, \mathrm{Ma}, \mathrm{Me}\end{array}$ & $\begin{array}{l}\text { Bodenheimer, 1949; Erkılıç and } \\
\text { Uygun 1995; Mohammed et al. } \\
2016\end{array}$ \\
\hline Salicicola archangelskyae (Lindinger) & C.monogyna, C. oxyacantha, $C$. orientalis, $F$. excelsior & $\begin{array}{l}\mathrm{Ae}, \mathrm{Ca}, \mathrm{Ea}, \\
\mathrm{Me}\end{array}$ & Yaşar, 1995; Kaydan et al., 2014 \\
\hline S. kermanensis (Lindinger) & Populus sp., P. nigra, Quercus sp., & $\mathrm{Ea}$ & Yaşar, 1995 \\
\hline Targioni vitis (Signoret) & A. hippocastaneum, Castanea crenata L., Quercus sp. & $\mathrm{Ca}, \mathrm{Ma}, \mathrm{Me}$ & Kaydan et al., 2013 \\
\hline \multicolumn{4}{|c|}{ Eriococcidae } \\
\hline $\begin{array}{l}\text { Eriococcus buxi (Boyer de } \\
\text { Fonscolombe) }\end{array}$ & Buxus sempervirens $\mathrm{L}$. & $\mathrm{Bs}, \mathrm{Ma}$ & $\begin{array}{l}\text { Kaydan and Kozár, 2008; } \\
\text { Ülgentürk et al., } 2008\end{array}$ \\
\hline \multicolumn{4}{|c|}{ Kermesidae } \\
\hline Kermes bacciformis Leonardi & Quercus sp., Q. coccifera & $\mathrm{Me}$ & Kaydan et al., 2013 \\
\hline Kermes sp. nr bacciformis Leonardi & Quercus sp. & $\mathrm{Ea}, \mathrm{Me}$ & Ülgentürk et al., 2013 \\
\hline K. bekiri Bodenheimer & Q. aegilops & $\mathrm{Ae}$ & Bodenheimer, 1953 \\
\hline K. greeni Bodenheimer & Q. coccifera, Q. ilex & $\mathrm{Ae}, \mathrm{Me}$ & Ülgentürk et al., 2013 \\
\hline
\end{tabular}


Table 2. Scale insects on broad-leaved trees, shrubs and other plants in Turkey (continued)

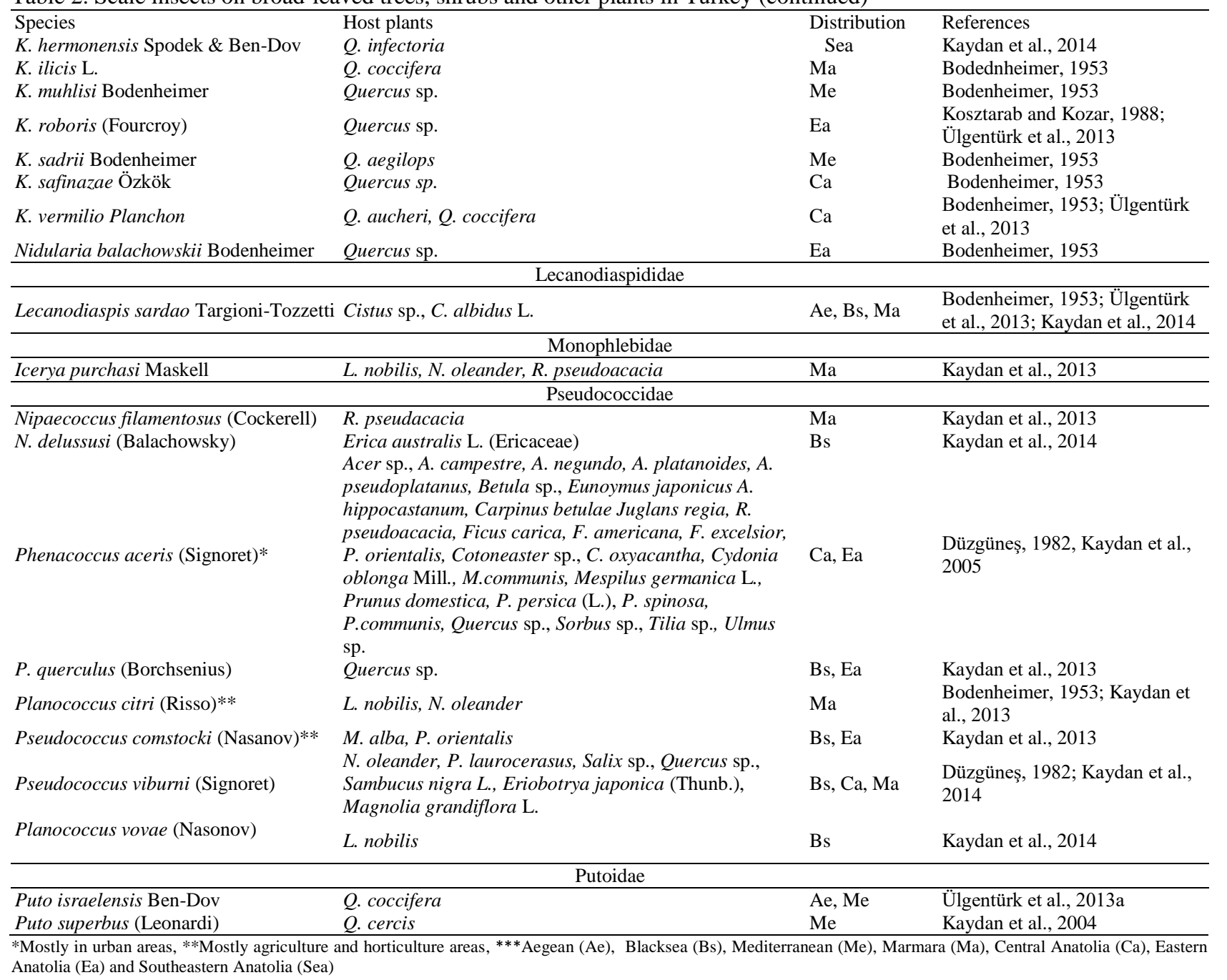

\subsection{Scale insects on conifer plants in Turkey}

About 42 species of scale insects on conifer trees were reported in Turkey (Kaydan et al., 2013; Keçe and Ulusoy, 2017; Ülgentürk et al., 2019a,b; Ülgentürk and Özdemir 2019). Pine trees vary according to climate and region. They also have the richest fauna of scale insects (twenty species). In forests of Turkey, Leucaspis pusilla Löw, Gomezmenornaspis cedricola Leonardi, M. hellenica, Matsucoccus josephi Bodenheimer and Harpaz (Matsucoccidae) and Paleococcus fuscipennis Burnmeister (Monophlebidae) are the most common species on pines, however Torosaspis cedricola (Balachowsky \& Alkan) on cedar trees (Ülgentürk et al., 2012a,b). Except Leucapis spp., the other species are fed on woody part of Conifer trees (Ülgentürk et al., 2012b). Although M. hellenica is accepted as useful since its honeydew is a resource of Pine honey, actually it is a phloem sap feeder and cause yellow spots, early falling of needles, die back branches and destroyed young pine trees (Ülgentürk et al., 2012b, Ülgentürk et al., 2013b). M. hellenica has one generation in a year and overwinters mostly third instar nymph in Aegean pine forest in Turkey (Bodenheimer, 1953; Gürkan, 2005, Ülgentürk et al., 2011). Many predators play an important role on plant health, through regulating $M$. hellenica populations. Among those predators Neoleucopis kartliana (Tanasijtshuk) (Diptera: Chamaemyiidae) is the most efficient and common predator of $M$. hellenica (Ülgentürk et al., 2013c). Recently, M. caucasica Hadzibejli was found on Abies nordmanniana and Piceae orientalis in Blacksea forest (Ülgentürk et al., 2019b). The Israel Pine Blast Scale $M$. josephi was recorded on P. brutia, P. halepensis and $P$. pinea in forest of southern and Western Anatolia (Ülgentürk et al., 2013c). Afterwards Ülgentürk et al. (2016) determined that $M$. josephi has at least 4 generations per year and overwinters as second stage (cyst) underneath the bark of pine trees. Male and female with eggs were observed four times in March-April, May-June, July-August and the end of September. The adult females were settled for oviposition on the lower stems at the base of needlebunches, on old cones and underneath the bark. Female produced a loose white ovisac and deposited 188-293 eggs. Elatophilus hebraicus Pericart (Hemiptera: Anthocoridae) is common and effective predator in Turkey (Ülgentürk, 2016). P. fuscipennis is a common monophlebid in Mediterranean and Aegean pine forests in Turkey (Ülgentürk et al., 2011). Like other monophlebids, it feeds on phoem and secretes honeydew (Ülgentürk et al., 2012a).

The range of cedar (Cedrus spp.) forest presently covers about 600,000 ha in Turkey (Boydak and Çalıkoğlu, 2008). 
Cedar trees are generally used for commercial purpose in Turkey. The most common and harmful pest of cedar is $T$. cedricola whereas totally 13 scale insect were recorded on cedar trees in Turkey (Ülgentürk et al., 2012a; Ülgentürk et al., 2013b). High population of $T$. cedricola has a very harmful effect on cedar trees, especially young cedar trees in urban green areas. High levels of sulphur dioxide and dust limit the activity of natural enemies in urban planting cedar (Dostbil, 2012). T. cedricola has 2 generations in a year and overwinters as fertilized female on needles of cedar (Dostbil and Ülgentürk, 2016). Dynaspidiotus britannicus (Newstead) (Diaspididae) feeds on cedar, fir and spruce species while $T$. cedricola occurs only cedar species in Turkey. D. britannicus has two generations and overwinters as second nymph instar on needle of cedar (Ayhan and Ülgentürk, 2011). Physokermes hellenicus Kozar and Gounari (Coccidae) is distributed both natural fir forest and urban belts. It hibernates as third nymph stage and has one generation in a year in urban areas of Ankara (Ülgentürk, 2016). On the other hand, Physokermes piceae (Schrank) is found only on urban spruce trees of Ankara, Eskişehir and İstanbul (Çanakçıŏlu, 1977; Ülgentürk et al., 2008). $P$. piceae is the main pest of spruce in parks and gardens of Ankara (Ülgentürk and Toros, 1999a; Ülgentürk et al., 2019a). It has one generation in a year and overwinters as second nymph stage (Turguter and Ülgentürk, 2006). Both species have potential of honeydew honey production in Turkey. Aphycoides clavallatus (Dalman) (Hymenoptera: Encyrtidae) is dominant parasitoids of $P$. piceae and $P$. hellenicus in Ankara. P. hellenicus is associated with a few number predators namely; Anthribus nebulosus Forster (Coleoptera: Anthribidae), Chilocorus renipustulatus Scriba (Coleoptera: Coccinellidae), Atroctotomus sp. (Hemiptera: Anthocoridae) in Turkey (Ülgentürk, 2001; 2016). Anthribus fasciatus Forster (Coleoptera: Anthribidae) was the most abundant natural enemy of $P$. hellenicus in Greece (Papanastasiou et al., 2018).

Phenacoccus piceae Löw (Pseudococcidae) has very large population on oriental spruce urban areas, while it is rare in natural forest of Blacksea region (Ülgentürk and Özdemir, 2019). About ten scale insect species are known on fir in Turkey. Eulecanium sericeum Lindinger, Nemolecanium abietis and M. caucasica are recently determined on fir in Blacksea Region (Ülgentürk et al., 2017). There are only five scale insect species that were recorded on juniper, among them Planococcus vovae (Hemiptera: Pseudococcidae) attracts notice by its honeydew production (Ülgentürk et al., 2013b).

A pest of yew trees [Taxus spp., (Taxaceae)] Pulvinaria floccifera (Westwood) (Coccidae) overwinters as the second nymph instar and has two generations per year in İstanbul. Chilocorus bipustulatus (L.) and Exochomus quadripustulatus (L.) (Coleoptera: Coccinellidae) are recorded as the predators and Coccophagus lycimnia (Walker) (Hymenoptera: Aphelinidae) as the parasitoid of P. floccifera (Selmi, 1979). Parthenolecanium pomeranicum (Kawecki) (Coccidae) is seldom scale insect on yew trees in Turkey (Ülgentürk and Toros, 1999a).

\subsection{Scale insects on broad-leaved trees and shrubs in Turkey}

Main broad-leaved trees of the Turkey forest are oak species (Anonymous, 2019). Over 40 scale insect species on oak trees were recorded in Turkey. Only 20 species are belonging to Asterolecaniidae (8) and Kermesidae (12) families, and are feeding only on oak trees (Table 2). First record of scale insect on oaks was Kermes ilicis L. (Kermesidae) on Quercus coccifera which is called as Kermes oak (Fahringer, 1922). K. ilicis were used commonly to dying silk and wool materials in Mediterranean and Middle East Regions in ancient times (Bodenheimer, 1953). Recently Kermes hermonensis Spodek \& Ben-Dov was detected on Quercus infectoria Oliv. in Diyarbakır (Kaydan et al., 2014). Beside chesnut and hazelnut, Parthenolecanium rufulum (Coccidae) is a pest of oaks in Turkey (Ecevit et al., 1987). It has one generation in a year and overwinters as second nymph instar on branches (Kaplan et al., 2016). Parthenolecanium corni (Bouché) (Coccidae) is polyphagous species that is common on fruits, ornamental and forest trees including oaks, beech, ash, maple, elm, polar and willow (Kaydan et al., 2013). On the other hand, Eulecanium ciliatum (Douglas) (Coccidae) is a widespread soft scale insect on Aceraceae (Acer campestre L., A. negundo L., Acer pseudoplatanus L.), and Rosaceae (Crateagus monogyna Jacq, C. oxyacantha L., Ribes sp., Cydonia sp.) in the parks and gardens of Ankara (Ülgentürk and Toros, 1999a). Following heavy infestations, branches dried up and sometimes the whole tree died. It has one generation per year and overwinters as second nymph stage on the host plants in Ankara (Ülgentürk and Toros, 1999c). The most common parasitoid of E. ciliatum was Encyrtus infidus (Rossi) (Hymenoptera: Encyrtidae) which made up of $66 \%$ of all parasitoids and the common predator was Anthribus fasciatus (Förster) Coleoptera: Anthribidae) in Ankara (Ülgentürk and Toros, 1999b). The mapple mealybug Phenacoccos aceris (Signoret) (Pseudococcidae) is a polyphagous species recorded mostly on urban plants in Turkey. It has one generation in a year and overwinters as third nymph stage in the bark and other hidden places on the plant. At the end of February and in early March, the nymphs started to move to the one year old twigs. The female started egg laying in the middle of April in both years. Hatching started at the beginning of the June and first-instars nymphs settled on the leaf and remained there until the end of September. After this period, the nymphs moved to the bark to overwinter. The population was affected by natural enemies and by environmental conditions throughout the year (Kaydan et al., 2006; Kaydanet al. 2015). This mealybug is supposed to be of European origin where occasionally becomes a pest on ornamental or fruit trees (Kosztarab and Kozár, 1988). $P$. aceris was verified as the vector of the Little Cherry Virus 2 (LChV-2) and GLRaV species, Grapevine virus A and Grapevine virus B (GVA and GVB) in Europe (Le Maguet et al., 2012; Garcia et al., 2016). Melanaspis inopinata Leonardi (Diaspididae) is a common pest on ash, horse chestnut and other park and forest plants (Ülgentürk and Toros, 1996; Kaydan et al., 2013). It has one generation annually, and overwinters as adult female in Ankara (Ayten and Ülgentürk, 2007). Kermes spp., P. rufulum, P. corni, Puto israelensis Ben-Dov are considered to be the sources of honeydew honey in oak forest in Turkey (Ülgentürk et al., 2013a, b).

Many species that are very common and serious pest on agriculture and horticulture plants are found on forest plants too. For example, olive scale insect Parlatoria oleae (Colveé) (Diaspididae) is an important pest on olive trees 
and apple, pear, quince and other species of Rosaceae. It has two generations in a year and overwinters as female in Turkey (Uygun et al., 2010). White peach scale Pseudaulacaspis pentagona (Targioni Tozzetti) (Diaspididae) is a pest of economic importance for mulberry, peach trees and woody ornamentals and it is widespread all the fruit-growing areas of Turkey (Yaşar, 1995; Uygun et al., 2010). It feeds on the trunks, branches and twings. In heavy infestations, dieback and death of trees occur (Erkılıç and Uygun, 1995). It is recorded on twentytwo host plant species in Ankara (Mohammed et al. 2016). Saissetia oleae (Olivier) (Coccidae) is poliphagous and important pest species on citrus and olive orchards. S. oleae is recorded on wild olive and oleander in maquies of Aegean and Mediterranean forests (Uygun et al., 2010). Planococcus citri (Risso) (Pseudococcidae) is the most cosmopolitan mealybug species that infested over 300 plant species in tropics and subtropics as well as in greenhouse (Williams, 2004). It is considered as one of the most major pest of citrus orchards in Turkey, due to the fact that its chemical control has not been achieved successfully yet. It has 3 generations per year and can be controlled using the parasitoid Leptomastix dactylopii Howard (Hymenoptera: Encyrtidae) and the predator Chryptolaemus montrouzieri Mulsant (Coleoptera: Coccinellidae) in citrus orchards in Turkey (Uygun et al., 2010). A few numbers of scale insects on shrubs are determined in forest areas. Rhodococcus perornatus (Cockerell \& Parrott) (Coccidae) is recorded on oil rose in horticulture and dog rose in forest areas (Ülgentürk et al., 1999). Lecanodiaspis sardoa Targioni Tozzetti (Leconaspidae) and Acanthococcus melnikensis (Hodgson \& Trencheva) (Acanthococcidae) had large populations on Cistus sp. (Cistaceae) and Myrtus spp. (Myrtaceae), respectively. These plants are occurred in sunny places in or border of forests (Aydınözü, 2008). Trabutina crassispinosa Borchsenius and T. mannipara (Hemprich \& Ehrenberg) (Pseudococcidae) are seldom scale insects while Prodiaspis tamaricicola (Malenotti) (Diaspididae) is very common on Tamarix spp. (Tamaricaceae) in Turkey (Kaydan et al. 2013; Kaydan and Kozar, 2008).

\section{Conclusions}

In this study is determined totally 137 scale insect in forest trees in Turkey. Among of them 43 scale insects [Coccidae (10), Diaspididae (22), Eriococcidae (1), Marchalinidae (2), Matsucoccidae (2), Monophlebidae (1) and Pseudococcidae (5)] are occured on conifer in Turkey. In addition, totally 95 scale insect species belonging to Acanthococcidae (8), Asterolecaniidae (8), Coccidae (18), Cryptococcidae (2), Diaspididae (34), Eriococcidae (1), Kermesidae (12), Lecanospidae (1) Monophlebidae (1), Psedocccidae (8) and Putoidae (2) are reported on broadleaved trees and shrubs in forest and urban areas in Turkey. Most of them infest agriculture and horticulture plants. Insects in forests and other natural areas have the potential to become a source of contamination for cultured plants. On the other hand, natural enemies that we can use against insect pests of cultivated plants can maintain themselves only in natural areas. In this regard, knowledge on destructive and beneficial species no doubt will help us understand, protect and use the ecosystem without destroying it.

\section{References}

Alkan, B., 1957. Tee scadlinge in der Türkei. Zeitshrift für Angewandte Entomologie, 41: 233-245.

Aydınözü, D., 2008. An investigation on the distribution areas of the maquis formation in Turkey. Kastamonu Eğitim Dergisi, 16(1): 207-220.

Ayhan, B., Ülgentürk, S., 2011. Phenology of Dynaspidiotus britannicus (Newstead) (Hemiptera: Diaspididae) on the cedar in Ankara. Procedings of the $4^{\text {th }}$ Plant Protection Congress of Turkey,28-30 Haziran, Kahramanmaraş, Turkey, pp.38.

Ayten, S., Ülgentürk, S., 2007. Morphology and biology of Melanaspis inopinata Leonardi (Hemiptera: Diaspididae) on firethorn (Pyracantha coccinea) in Turkey. Proceeding of the XI International Symposium on Scale Insect Studies, 24-27 September, Oeiras, Portugal, pp. 213-216.

Ben-Dov, Y., Gounari, S., Kaydan, M.B., Hadina, F., 2006. Phenacoccus yerushalmi Ben-Dov newly recorded from Greece and Turkey (Hem., Coccoidea, Pseudococcidae). Bulletin de la Société Entomologique de France, 111(1): 42.

Bodenheimer, F.S., 1949. The Coccoidea of Turkey. Diaspididae. A monographic study. Güney Matbaacılık ve Gazetecilik T.A.O., Ankara.

Bodenheimer, F.S., 1952. The Coccoidea of Turkey. I. Revue de la Faculté des Sciences de L'Université D'Istanbul, (Serie. B), 17: 315-351.

Bodenheimer, F.S., 1953. The Coccoidea of Turkey. III. Revue de la Faculté des Sciences de L'Université D'Istanbul, (Série. B), 8: 91-164.

Boydak, M., Çalıkoğlu, M., 2008. Biology and Silviculture of Lebanon Cedar (Cedrus libani A. Rich.). OGEM-VAK. Lazer Ofset Press, Ankara.

Çanakçığlu, H., 1977. Türkiye'de Orman Ağaçları ve Ağaççıklarında Zarar Yapan Coccoidea (Hom.) Türleri Üzerinde Araştırmalar (Sistematik-Yayılış-Konukçu-Biyoloji). İstanbul Üniversitesi, Orman Fakültesi Yayınları: 2322, İstanbul.

Dostbil Sahin, Ö., 2012. Distribution of cedar scale insect Torosaspis (Acanthomytilus) cedricola (Balachowsky \& Alkan) (Hemiptera: Diaspididae) in Turkey and its bio-ecology in Ankara. PhD Dissertation, Ankara Üniversity, Institute of Natural and Applied Sciences, Ankara.

Dostbil, Ö., Ülgentürk, S., 2016. Bio-ecology of cedar scale insect Torosaspis cedricola (Balachowsky \& Alkan) (Hemiptera: Diaspididae) in Ankara, Turkey. Redia, 99: 163-170.

Düzgüneş, Z., 1982. Studies on Pseudococcidae (Homoptera: Coccoidea) Species of Turkey. Ankara Üniversitesi Ziraat Fakültesi Yayınları No: 836, Ankara.

Ecevit, O., Işık, M., Yılmaz, F., 1987. Fındıklarda Zararlı Fındık Koşnili Parhenolecanium corni ve P.rufulum ile Virgül Kabuklu Biti Lepidosaphes ulmi'nin Biyoekolojik Özellikleri ve Fındık Koşnilinin Mücadele Metotları Üzerine Araştırmalar. 19 Mayıs Üniversitesi yayınları, No: 19, Samsun.

Erkılıç, L., Uygun, N., 1995. Distribution, population fluctuations and natural enemies of the white peach scale, Pseudaulacaspis pentagona (Targioni-Tozzetti) (Homoptera:Diaspididae) in the East mediterranean region of Turkey. Israel Journal of Entomology, 29: 191-198.

Erkı1ıç, L.B., Kaydan, M.B., Kozár, F., 2011. Description of a new species of Eriococcidae (Hemiptera: Coccoidea) from Turkey. Turkish Journal of Zoology, 35(1): 15-22.

Erözmen, K., Yaşar, B., 2018. Determination of Diaspididae species (Hemiptera: Coccomorpha) on Fruit trees in Balıkesir. Süleyman Demirel University Journal of Natural and Applied Sciences, 22(1): 172-181.

FAO. 2019. Türkiye'nin Biyoçeşitliliği: Genetik Kaynakların Sürdürülebilir Tarım ve Gıda Sistemlerine Katkısı. Ankara, Licence: CC BY-NC-SA 3.0 IGO. 
Foldi, I., 2004. The Matsucoccidae in the Mediterranean basin with a World list of species (Hemiptera: Sternorrhyncha: Coccoidea). Annales de la Société entomologique de France, 40: 145-168.

García, M.M., Denno, B.D., Miller, D.R., Miller, G.L., Ben-Dov, Y., Hardy, N.B., 2016. ScaleNet: A literature-based model of scale insect biology and systematics. Database. Available from: http://scalenet.info Accessed:10.10.2019.

Gürkan, B., 2005. Studies on life cycle characteristics of Marchalina hellenica Genn. (Hemiptera: Margarodidae) in areas with different altitudes. Mellifera, 5(9): 2-6.

Houston, D.R., 1994. Temporal and spatial shift within the Nectria pathogen complex associated with beech bark disease of Fagus grandifolia. Canadian Journal of Forest Research, 24(5): 960968.

Kaplan, C., Turanl1, T., 2016. Determination of distribution, biology and natural enemies of Parthenolecanium rufulum (Cockerell) (Hemiptera: Coccidae) on chestnut trees in İzmir and Manisa Provinces in Turkey. Journal of Turkish Entomology, 40(3): 331-343

Kaydan, M.B., Kılınçer, N., Uygun, N., Japosvili, G., Gaimari, S., 2006. Parasitoids and predators of Psedococcidae (HomF Hodgsonoptera: Coccoidea) in Ankara, Turkey. Phytoparasitica, 34(4): 331-337.

Kaydan, M. B., Kılınçer, A N., Kondo, T., 2015. Descriptions of all female stages of the maple mealybug, Phenacoccus aceris (Hemiptera: Coccoidea: Pseudococcidae), with notes on its biology. Acta Zoologica Academie Scientiarum Hungaricae, 61 (3): 255-277. Kaydan, M.B., Kozár, F., 2008. Two new genera and species of Eriococcidae (Hemiptera: Sternorrhyncha: Coccoidea) with new data on the family in Turkey. Zootaxa, 1848: 16-26.

Kaydan, M.B., Kozár, F., 2010. A review of the genus Neoacanthococcus Borchsenius (Hemiptera: Coccoidea: Eriococcidae) with a description of Neoacanthococcus atlihani sp. nov. in Turkey. Turkiye Entomoloji Dergisi, 34(2): 165-177.

Kaydan, M.B., Ülgentürk, S., Erkılıç, L., 2013. Checklist of Turkish Coccoidea (Hemiptera: Sternorrhyncha) species. Bulletin of Turkis Entomology, 3(4): 157-182.

Kaydan, M.B., Bolu, H., Spodek, M., Ben-Dov, Y., Tuğrul, A.F., 2014. The first record of Kermes hermonensis Spodek \& BenDov (Hemiptera: Sternorrhyncha: Coccoidea: Kermesidae) in Turkey. Journal of Entomological Research Society, 16(3): 9599.

Kaydan, M.B., Ülgentürk, S., Özdemir, I., Ulusoy, M.R., 2014. Coccoidea (Hemiptera) species in Bartın and Kastamonu Provinces. Bulletin of Turkis Entomology, 54(1): 11-44.

Keçe-Çalışkan, A.F., Ulusoy, M.R., 2017. Armored scale insects (Hemiptera: Sternorrhyncha: Diaspididae) on ornamental plants in Adana, Turkey. Journal of Turkish Entomology, 41(3): 333-346.

Kosztarab, M., Kozár, F., 1988. Scale Insects of Central Europe. Akademiai Kiado, Budapest.

Kozár, F., Kaydan, M.B., Konczné Benedicty, Z., Szita, É., 2013. Acanthococcidae and Related Families of the Palaearctic Region. Hungarian Academy of Sciences, Budapest.

Le Maguet, J., Beuve, M., Herrbach, E., Lemaire, O., 2012. Transmission of Six Ampeloviruses and two vitiviruses to grapevine by Phenacoccus aceris. Virology, 102(7): 717-723.

Mohammed, A.M.E., Ülgentürk, S., Uygun, N., Garonna, A.P., Szenkiraly, F., Fent, M., Hayat, M., 2016. The distribution, host plants and natural enemies of White peach scale, Pseudaulacaspis pentagona (Targioni-Tozzetti) in Ankara Province. Munis Entomology \& Zoology, 11(2): 650-656.

Papanastasiou, I., Kavallieratos, N.G., Saitanis, C.J., Chatzaki, M., Georgios, Th., Papadoulis, G. Th.G., 2018. Parasitoids and predators of physokermes hellenicus (Hemiptera: Coccomorpha: Coccidae) in Greece. Journal of Economic Entomology, 111(3): 1121-1130.
Özkazanç, O., Yücel, M., 1985. Reserach on Harmful Insects of Semi-Arid Region Plantations. Journal of Forestry Research Institute, Technical Bulletin Series No: 153, Ankara.

Selmi, E., 1979. Researches Pest Coccoidea (Homoptera) species of Conifer Trees in Marmara Region (systematic- distributionhost-biology-Natural enemies). İ.Ü. Orman Fakültesi Dergisi, Seri A., 29: 92-127.

Schmitschek, E., 1953. Forstinsekten der Türkei und ihre Umwelt.: Hüsnütabiat Matbaası, Istanbul.

Şahin, Ö., Ülgentürk, S., 2011. Distribution of Torasaspis (Acanthomytilus) cedricola Balachowsky\& Alkan (Hemiptera: Diaspididae) in the Turkey. I. Forest Entomology and Pathology Symposium of Turkey, 23-25 Nowember, Antalya. pp. 261-265.

TOD, 2019. Türkiye Ormancılığgi:2019. Türkiye Ormancılar derneği, yayın No:47 Kuban Matbaacılık Yayıncılık, Ankara.

Turguter, S., Ülgentürk, S., 2006. Physokermes piceae (Schrank) (Yumrulu Ladin Koşnili) (Hemiptera; Coccidae)'nin biyolojik özellikleri. Tarım Bilim Dergisi, 12: 44-50.

Ülgentürk, S., Toros, S., 1999a. Faunistic studies on Coccidae from ornamental plants in Ankara, Turkey. Entomologica, 33: 213217.

Ülgentürk, S., Toros, S., 1999b. Natural enemies of Oak scale Insect, Eulecanium ciliatum (Douglas) (Hemiptera: Coccidae) in Turkey. Entomologica, 33: 219-224.

Ülgentürk, S., Toros, S., 1999c. Studies on the biology of Eulecanium ciliatum (Douglas) (Hemiptera: Coccidae) in Turkey. Entomologica, 33: 351-356.

Ülgentürk, S., 2001. Parasitoids and predators of Coccidae Homoptera: Coccoidea) species on ornamental plants in Ankara, Turkey. Acta Phytopathologica et Entomologica Hungarica, 36(3-4): 369-375.

Ülgentürk, S., Çanakçığlu, H., Toper, A. 2004. Scale insects of the conifer trees in Turkey and their zoogeographical distribution. Journal of Pest Science, 77: 99-104.

Ülgentürk, S., Çanakçığlu, H., 2004. Scale insect pests on ornamental plants in urban habitats in Turkey. Journal of Pest Science, 77: 79-84.

Ülgentürk, S., Şahin, Ö., Kaydan, M.B., 2008. Coccoidea (Hemiptera) species on park plants in urban areas of Istanbul province. Bitki Koruma Bülteni, 48: 1-18.

Ülgentürk, S., Ayhan, B., 2011. Türkiye tarımında yeni bir zararlı; Neopulvinaria innumerabilis (Rathvon) (Hemiptera: Coccidae). Türkiye Entomoloji Bülteni, 1(3): 189-195.

Ülgentürk, S., Dursun, O., Ayhan, B., Şahin, Ö., Evren, N., 2011. Distribution and phenology of Palaeococcus fuscipennis (Burnmeister) (Hemiptera: Monophlebidae) in Aegean and Mediterraean Regions. (as abstract). I. Forest Entomology and Pathology Symposium of Turkey, 23-25 Nowember, Antalya. pp. 272.

Ülgentürk, S., Civelek, H.S., Evren, N., Şahin Dostbil, Ö., Sarıbaşak, H., 2012a. Çam Pamuklu Koşnili Marchalina hellenica Genn. (Hemiptera: Margarodidae)'nın Biyo-Ekolojisi, Ege ve Akdeniz Bölge'sindeki Yayılış Alanları. TUBİTAK Proje Nihai Raporu, Ankara.

Ülgentürk, S., Şahin, Ö., Ayhan, B., Sarıbaşak, H., Kaydan, M.B., 2012b. Coccoidea (Hemiptera) species of Taurus cedar (Cedrus libani) in Turkey. Türkiye Entomoloji Dergisi, 6: 113-121.

Ülgentürk, S., Evren, N., Ayhan, A., Dostbil, Ö., Dursun, O., Civelek, H.S., 2012c. Scale insect (Hemiptera: Coccoidea) species on pine trees of Turkey. Journal Turkish Zoology, 36: 623-636.

Ülgentürk, S., Kaydan, M.B., Kozár, F., Ben-Dov, Y., 2013a. Coccoidea (Hemiptera) species on oaks in Turkey. Türkiye Entomoloji Bülteni, 3: 13-31.

Ülgentürk, S., Özdemir, I., Kozár, F., Kaydan, M.B., Dostbil, Ö., Sarıbaşak, H., Civelek, H.S., 2013b. Honey producing insect species in forest areas in Wetern Turkey. Türkiye Entomoloji Bülteni, 3: 125-133. 
Ülgentürk, S., Szentkirályi, F., Uygun, N., Fent, M., Gaimari, S.D., Civelek, H., Ayhan, B., 2013c. Predators of Marchalina hellenica (Hemiptera: Marchalinidae) on pine forests in Turkey. Phytoparasitica, 41: 529-537.

Ülgentürk, S., 2016. Biology, natural enemies and distribution of Physokermes hellenicus Kozár \& Gounari (Hemiptera: Coccidae) in Turkey. Entomologica, 47: 71-75.

Ülgentürk, S., Fent, M., Civelek, S.H., 2016. Biological observations of Matsucoccus josephi (Hemiptera: Matsucoccidae) in Turkey. Entomologica, 47: 67-70.

Ülgentürk, S., Özdemir, M., 2019 Scale insects Hemiptera: Coccomorpha: Coccoidea) on Oriental spruce in Turkey. Turkish Journal of Forestry, 20(2): 76-79.

Ülgentürk, S., Özdemir, I., Muştu, M., Dostbil, Ö., Erbaş, C. 2019a. Pest species of Aphidomorpha and Coccomorpha (Hemiptera) on conifers urban areas of Ankara, Turkey. Munis Entomology \& Zoology, 14: 51-61.

Ülgentürk, S., Cosic, B., Özdemir, I., İpek, A., Sorkun, K., 2019 b. Insects as the source of honeydew honey in some cedar, fir, oak, spruce forests of Turkey. Baltic Forestry, 25 (in press).
Uygun, N., Ulusoy, M.R., Karaca, İ., Satar, S., 2010. Pests of Orchard and Vineyard. Özyurt Matbaacılık, Adana.

Williams, D.J., 2004. Mealybugs of Southern Asia. The Natural History Museum Kuala Lumpur: Southdene SDN. BHD.

Yaşar, B., 1990. Coccidae ve Diaspididae (Hom.; Coccoidea) species on ornamental plants in İzmir. PhD Dissertation, Ege University, Institute of Natural and Applied Sciences, İzmir, Turkey.

Yaşar, B., 1991. Türkiye faunası için yeni bir Asterodiaspis signoret (Homoptera, Asterolecaniidae) türü. Türkiye Entomoloji Dergisi, 15(1): 61-64.

Yaşar, B., 1995. Türkiye Diaspididae (Homoptera: Coccoidea) Faunas1 Üzerinde Taksonomik Araştırmalar. Yüzüncü Y1l Üniversitesi Matbaası, Van

Zeki, C., Ülgentürk, S., Kaydan, M.B., Özmen, D., Toros, S., 2005. Records of scale insects (Hemiptera: Coccoidea) from orchards and neighbouring plants in provinces Afyon, Ankara, Burdur, Isparta, Turkey. Proceeding of the International Symposium on Scale Insect Studies, 19-23 April, Adana, Turkey, pp.185-196. 